\title{
Avoiding maximal parabolic subgroups of $S_{k}$
}

\author{
Toufik Mansour ${ }^{1 \dagger}$ and Alek Vainshtein ${ }^{2}$ \\ Department of Mathematics and Department of Computer Science, University of Haifa, Haifa, Israel 31905. \\ ${ }^{1}$ Email: tmansurestudy.haifa.ac.il ${ }^{2}$ Email: alek@mathcs.haifa.ac.il
}

received 4 Jul 2000, accepted 16 Nov 2000.

We find an explicit expression for the generating function of the number of permutations in $S_{n}$ avoiding a subgroup of $S_{k}$ generated by all but one simple transpositions. The generating function turns out to be rational, and its denominator is a rook polynomial for a rectangular board.

Keywords: permutations, forbidden patterns, parabolic subgroups, Laguerre polynomials, rook polynomials

\section{Introduction and Main Result}

Let $[p]=\{1, \ldots, p\}$ denote a totally ordered alphabet on $p$ letters, and let $\alpha=\left(\alpha_{1}, \ldots, \alpha_{m}\right) \in\left[p_{1}\right]^{m}$, $\beta=\left(\beta_{1}, \ldots, \beta_{m}\right) \in\left[p_{2}\right]^{m}$. We say that $\alpha$ is order-isomorphic to $\beta$ if for all $1 \leq i<j \leq m$ one has $\alpha_{i}<\alpha_{j}$ if and only if $\beta_{i}<\beta_{j}$. For two permutations $\pi \in S_{n}$ and $\tau \in S_{k}$, an occurrence of $\tau$ in $\pi$ is a subsequence $1 \leq i_{1}<i_{2}<\ldots<i_{k} \leq n$ such that $\left(\pi_{i_{1}}, \ldots, \pi_{i_{k}}\right)$ is order-isomorphic to $\tau$; in such a context $\tau$ is usually called the pattern. We say that $\pi$ avoids $\tau$, or is $\tau$-avoiding, if there is no occurrence of $\tau$ in $\pi$. Pattern avoidance proved to be a useful language in a variety of seemingly unrelated problems, from stack sorting [Kn, Ch. 2.2.1] to singularities of Schubert varieties [LS]. A natural generalization of single pattern avoidance is subset avoidance; that is, we say that $\pi \in S_{n}$ avoids a subset $T \subset S_{k}$ if $\pi$ avoids any $\tau \in T$. The set of all permutations in $S_{n}$ avoiding $T \subset S_{k}$ is denoted $S_{n}(T)$. A complete study of subset avoidance for the case $k=3$ is carried out in [SS]. For $k>3$ the situation becomes more complicated, as the number of possible cases grows rapidly. Recently, several authors have considered the case of general $k$ when $T$ has some nice algebraic properties. Paper [BDPP] treats the case when $T$ is the centralizer of $k-1$ and $k$ under the natural action of $S_{k}$ on $[k]$ (see also Sec. 3 for more detail). In [AR], $T$ is a Kazhdan-Lusztig cell of $S_{k}$, or, equivalently, the Knuth equivalence class (see [St, vol. 2, Ch. A1]). In this paper we consider the case when $T$ is a maximal parabolic subgroup of $S_{k}$.

Let $s_{i}$ denote the simple transposition interchanging $i$ and $i+1$. Recall that a subgroup of $S_{k}$ is called parabolic if it is generated by $s_{i_{1}}, \ldots, s_{i_{r}}$. A parabolic subgroup of $S_{k}$ is called maximal if the number of its generators equals $k-2$. We denote by $P_{l, m}$ the (maximal) parabolic subgroup of $S_{l+m}$ generated by $s_{1}, \ldots, s_{l-1}, s_{l+1}, \ldots, s_{l+m-1}$, and by $f_{l, m}(n)$ the number of permutations in $S_{n}$ avoiding all the patterns in $P_{l, m}$. In this note we find an explicit expression for the generating function of the sequence $\left\{f_{l, m}(n)\right\}$.

\footnotetext{
${ }^{\dagger}$ Partially supported by HIACS.

1365-8050 (c) 2000 Maison de l'Informatique et des Mathématiques Discrètes (MIMD), Paris, France
} 
To be more precise, we prove the following more general result. Let us denote $\sigma=s_{1} s_{2} \ldots s_{k-1}$, that is, $\sigma=(2,3, \ldots, k, 1)$ (written in one-line notation), and let $a$ be an integer, $0 \leq a \leq k-1$ (here and in what follows $k=l+m)$. We denote by $f_{l, m}^{a}(n)$ the number of permutations in $S_{n}$ avoiding the left coset $\sigma^{a} P_{l, m}$; in particular, $f_{l, m}^{0}(n)$ coincides with $f_{l, m}(n)$. Let $F_{l, m}^{a}(x)$ denote the generating function of $\left\{f_{l, m}^{a}(n)\right\}$,

$$
F_{l, m}^{a}(x)=\sum_{n \geq 0} f_{l, m}^{a}(n) x^{n} .
$$

Recall that the Laguerre polynomial $L_{n}^{\alpha}(x)$ is given by

$$
L_{n}^{\alpha}(x)=\frac{1}{n !} e^{x} x^{-\alpha} \frac{d^{n}}{d x^{n}}\left(e^{-x} x^{n+\alpha}\right),
$$

and the rook polynomial of the rectangular $s \times t$ board is given by

$$
R_{s, t}(x)=s ! x^{s} L_{s}^{t-s}\left(-x^{-1}\right)
$$

for $s \leq t$ and by $R_{s, t}(x)=R_{t, s}(x)$ otherwise (see [R1, Ch. 7.4]).

Main Theorem. Let $\lambda=\min \{l, m\}, \mu=\max \{l, m\}$, then

$$
F_{l, m}^{a}(x) R_{l, m}(-x)=\sum_{r=0}^{\lambda-1} x^{r} r ! \sum_{j=0}^{r}(-1)^{j} \frac{\left(\begin{array}{l}
l \\
j
\end{array}\right)\left(\begin{array}{c}
m \\
j
\end{array}\right)}{\left(\begin{array}{l}
r \\
j
\end{array}\right)}+(-1)^{\lambda} x^{\lambda} \lambda ! \sum_{r=0}^{\mu-\lambda-1} x^{r} r !\left(\begin{array}{c}
\mu-r-1 \\
\lambda
\end{array}\right),
$$

or, equivalently,

$$
F_{l, m}^{a}(x)=\sum_{r=0}^{k-1} x^{r} r !-\frac{(-1)^{\lambda} x^{\mu}}{\lambda ! L_{\lambda}^{\mu-\lambda}\left(x^{-1}\right)} \sum_{r=0}^{\lambda-1}(k+r) ! x^{r} \sum_{j=r+1}^{\lambda}(-1)^{j} \frac{\left(\begin{array}{c}
l \\
j
\end{array}\right)\left(\begin{array}{c}
m \\
j
\end{array}\right)}{\left(\begin{array}{c}
k+r \\
j
\end{array}\right)},
$$

where $k=l+m=\lambda+\mu$.

The proof of the Main Theorem is presented in the next section.

As a corollary we immediately get the following result (see [Ma, Theorem 1]).

Corollary 1 Let $0 \leq a \leq k-1$, then

$$
f_{1, k-1}^{a}(n)= \begin{cases}(k-2) !(k-1)^{n+2-k} & \text { for } n \geq k \\ n ! & \text { for } n<k .\end{cases}
$$

Proof. Since $R_{1, k-1}=1+(k-1) x$, the Main Theorem implies

$$
F_{1, k-1}^{a}(x)=\frac{1-\sum_{r=0}^{k-3} x^{r+1}(k-r-2) r !}{1-(k-1) x}=\frac{x^{k-2}(k-2) !}{1-(k-1) x}+\sum_{r=0}^{k-3} x^{r} r !
$$

and the result follows.

Another immediate corollary of the Main Theorem gives the asymptotics for $f_{l, m}^{a}(n)$ as $n \rightarrow \infty$.

Corollary 1.2. $f_{l, m}^{a}(n) \sim c \gamma^{n}$, where $c$ is a constant depending on $l$ and $m$, and $\gamma$ is the maximal root of $L_{\lambda}^{\mu-\lambda}$; in particular, $\gamma \leq k-2+\sqrt{1+4(l-1)(m-1)}$.

Proof. Follows from standard results in the theory of rational generating functions (see e.g. [St, vol. 1, Ch. 4]) and the fact that all the roots of Laguerre polynomials are simple (see [S7, Ch. 3.3]). The upper bound on $\gamma$ is obtained in [II]. 


\section{Proofs}

First of all, we make the following simple, though useful observation.

Lemma 2 For any natural $a, l, m, n$ such that $1 \leq a \leq l+m-1$ one has $f_{l, m}^{a}(n)=f_{m, l}^{m+l-a}(n)$.

Proof. Denote by $\rho_{n}$ and $\kappa_{n}$ the involutions $S_{n} \rightarrow S_{n}$ that take $\left(i_{1}, i_{2}, \ldots, i_{k}\right)$ to $\left(i_{k}, \ldots, i_{2}, i_{1}\right)$ (reversal) to $\left(n+1-i_{1}, n+1-i_{2}, \ldots, n+1-i_{n}\right)$ (complement), respectively. It is easy to see that for any $T \subset S_{k}$, the involutions $\rho_{n}$ and $\kappa_{n}$ provide natural bijections between the sets $S_{n}(T)$ and $S_{n}\left(\rho_{k} T\right)$, and between $S_{n}(T)$ and $S_{n}\left(\kappa_{k} T\right)$, respectively. It remains to note that $\rho_{k} \kappa_{k} \sigma^{a} P_{l, m}=\sigma^{l+m-a} P_{m, l}$.

From now on we assume that $a \geq 0, l \geq 1, m \geq 1$ are fixed, and denote $b=n-m+a$. It follows from Lemma 2 that we may assume that $a \leq m$, and hence $b \leq n$. This means, in other words, that $\tau \in S_{k}$ belongs to $\sigma^{a} P_{l, m}$ if and only if $\left(\tau_{1}, \ldots, \tau_{l}\right)$ is a permutation of the numbers $a+1, \ldots, a+l$. In what follows we usually omit the indices $a, l, m$ whenever appropriate; for example, instead of $f_{l, m}^{a}(n)$ we write just $f(n)$.

For any $n \geq k$ and any $d$ such that $1 \leq d \leq n$, we denote by $g_{n}\left(i_{1}, \ldots, i_{d}\right)=g_{n ; l, m}^{a}\left(i_{1}, \ldots, i_{d}\right)$ the number of permutations $\pi \in S_{n}\left(\sigma^{a} P_{l, m}\right)$ such that $\pi_{j}=i_{j}$ for $j=1, \ldots, d$. It is natural to extend $g_{n}$ to the case $d=0$ by setting $g_{n}(\emptyset)=f(n)$.

The following properties of the numbers $g_{n}\left(i_{1}, \ldots, i_{d}\right)$ can be deduced easily from the definitions.

\section{Lemma 3}

(i) Let $n \geq k$ and $1 \leq i \leq n$, then

$$
g_{n}(\ldots, i, \ldots, i, \ldots)=0 .
$$

(ii) Let $n \geq k$ and $a+1 \leq i_{j} \leq b$ for $j=1, \ldots, l$, then

$$
g_{n}\left(i_{1}, \ldots, i_{l}\right)=0 .
$$

(iii) Let $n \geq k, 1 \leq r \leq d \leq l$, and $a+1 \leq i_{j} \leq b$ for $j=1, \ldots, d, j \neq r$, then

$$
g_{n}\left(i_{1}, \ldots, i_{d}\right)= \begin{cases}g_{n-1}\left(i_{1}-1, \ldots, i_{r-1}-1, i_{r+1}-1, \ldots, i_{d}-1\right) & \text { if } 1 \leq i_{r} \leq a \\ g_{n-1}\left(i_{1}, \ldots, i_{r-1}, i_{r+1}, \ldots, i_{d}\right) & \text { if } b+1 \leq i_{r} \leq n .\end{cases}
$$

Proof. Property (ii) is evident. Let us prove (1ii). By (Ii), we may assume that the numbers $i_{1}, \ldots, i_{l}$ are distinct. Take an arbitrary $\pi \in S_{n}$ such that $\pi_{j}=i_{j}$ for $j=1, \ldots, l$. Evidently, for any $r \leq a$ there exists a position $j_{r}>l$ such that $\pi_{j_{r}}=r$; the same is true for any $r \geq b+1$. Therefore, the restriction of $\pi$ to the positions $1,2, \ldots, l, j_{1}, j_{2}, \ldots, j_{a}, j_{b+1}, j_{b+2}, \ldots, j_{n}$ (in the proper order) gives an occurrence of $\tau \in \sigma^{a} P_{l, m}$ in $\pi$. Hence, $\pi \notin S_{n}\left(\sigma^{a} P_{l, m}\right)$, which means that $g_{n}\left(i_{1}, \ldots, i_{l}\right)=0$.

To prove (iii), assume first that $1 \leq i_{r} \leq a$. Let $\pi \in S_{n}$ and $\pi_{j}=i_{j}$ for $j=1, \ldots, d$. We define $\pi^{*} \in S_{n-1}$ by

$$
\pi_{j}^{*}= \begin{cases}\pi_{j}-1 & \text { for } 1 \leq j \leq r-1, \\ \pi_{j+1}-1 & \text { for } j \geq r \text { and } \pi_{j+1}>i_{r}, \\ \pi_{j+1} & \text { for } j \geq r \text { and } \pi_{j+1}<i_{r} .\end{cases}
$$

We claim that $\pi \in S_{n}\left(\sigma^{a} P_{l, m}\right)$ if and only if $\pi^{*} \in S_{n-1}\left(\sigma^{a} P_{l, m}\right)$. Indeed, the only if part is trivial, since any occurrence of $\tau \in \sigma^{a} P_{l, m}$ in $\pi^{*}$ immediately gives rise to an occurrence of $\tau$ in $\pi$. Conversely, any 
occurrence of $\tau$ in $\pi$ that does not include $i_{r}$ gives rise to an occurrence of $\tau$ in $\pi^{*}$. Assume that there exists an occurrence of $\tau$ in $\pi$ that includes $i_{r}$. Since $r \leq d \leq l$, this occurrence of $\tau$ contains $a$ entries that are situated to the right of $i_{r}$ and are strictly less than $i_{r}$. However, the whole $\pi$ contains only $a-1$ such entries, a contradiction. It now follows from (1) that property (11ii) holds for $1 \leq i_{r} \leq a$. The case $b+1 \leq i_{r} \leq n$ is treated similarly with the help of the transformation $\left(\pi \in S_{n}\right) \mapsto\left(\pi^{\circ} \in S_{n-1}\right)$ given by

$$
\pi_{j}^{\circ}= \begin{cases}\pi_{j} & \text { for } 1 \leq j \leq r-1, \\ \pi_{j+1}-1 & \text { for } j \geq r \text { and } \pi_{j+1}>i_{r}, \\ \pi_{j+1} & \text { for } j \geq r \text { and } \pi_{j+1}<i_{r} .\end{cases}
$$

Now we introduce the quantity that plays the crucial role in the proof of the Main Theorem. For $n \geq k$ and $1 \leq d \leq l$ we put

$$
A(n, d)=A_{l, m}^{a}(n, d)=\sum_{i_{1}, \ldots, i_{d}=a+1}^{b} g_{n}\left(i_{1}, \ldots, i_{d}\right) .
$$

As before, this definition is extended to the case $d=0$ by setting

$$
A(n, 0)=g_{n}(\emptyset)=f(n) .
$$

Theorem 4 Let $n \geq k+1$ and $1 \leq d \leq l-1$, then

$$
A(n, d+1)=A(n, d)-(m-d) A(n-1, d)-d A(n-1, d-1) .
$$

Proof. First of all, we introduce two auxiliary sums:

$$
\begin{aligned}
& B(n, d)=B_{l, m}^{a}(n, d)=\sum_{i_{1}, \ldots, i_{d}=a+1}^{b+1} g_{n}\left(i_{1}, \ldots, i_{d}\right), \\
& C(n, d)=C_{l, m}^{a}(n, d)=\sum_{i_{1}, \ldots, i_{d}=a}^{b} g_{n}\left(i_{1}, \ldots, i_{d}\right),
\end{aligned}
$$

where $b=n-m+a$; once again, $B(n, 0)=C(n, 0)=f(n)$.

Let us prove three simple identities relating together the sequences $\{A(n, d)\},\{B(n, d)\},\{C(n, d)\}$.

Lemma 5 Let $n \geq k$ and $1 \leq d \leq l$, then:

$$
\begin{gathered}
A(n, d)=A(n, d-1)-(m-a) B(n-1, d-1)-a C(n-1, d-1), \\
(m-a) A(n, d)=(m-a) B(n, d)-(m-a) d B(n-1, d-1), \\
a A(n, d)=a C(n, d)-a d C(n-1, d-1) .
\end{gathered}
$$

Proof. To prove the first identity, observe that by definitions and Lemma 3 (iiii) for the case $r=d$, one has

$$
A(n, d-1)-A(n, d)=\sum_{i_{1}, \ldots, i_{d-1}=a+1}^{b} \sum_{i_{d}=1}^{n} g_{n}\left(i_{1}, \ldots, i_{d}\right)-A(n, d)
$$




$$
\begin{aligned}
& =\sum_{i_{1}, \ldots, i_{d-1}=a+1}^{b}\left(\sum_{i_{d}=1}^{a} g_{n}\left(i_{1}, \ldots, i_{d}\right)+\sum_{i_{d}=b+1}^{n} g_{n}\left(i_{1}, \ldots, i_{d}\right)\right) \\
& =\sum_{i_{1}, \ldots, i_{d-1}=a+1}^{b}\left(a g_{n-1}\left(i_{1}-1, \ldots, i_{d-1}-1\right)+(m-a) g_{n-1}\left(i_{1}, \ldots, i_{d-1}\right)\right) \\
& =a C(n-1, d-1)+(m-a) B(n-1, d-1),
\end{aligned}
$$

and the result follows.

The second identity is trivial for $a=m$, so assume that $0 \leq a \leq m-1$ and observe that by definitions and Lemma 3 (1ii) and (iii), one has

$$
\begin{aligned}
B(n, d) & =\sum_{i_{1}, \ldots, i_{d}=a+1}^{b} g_{n}\left(i_{1}, \ldots, i_{d}\right)+\sum_{j=1}^{d}\left(\sum_{i_{1}, \ldots, \hat{\imath}_{j}, \ldots, i_{d}=a+1}^{b} g_{n}\left(i_{1}, \ldots, i_{j-1}, b+1, i_{j+1} \ldots, i_{d}\right)\right) \\
& =A(n, d)+\sum_{j=1}^{d}\left(\sum_{i_{1}, \ldots, \hat{\imath}_{j}, \ldots, i_{d}=a+1}^{b} g_{n-1}\left(i_{1}, \ldots, i_{j-1}, i_{j+1}, \ldots, i_{d}\right)\right) \\
& =A(n, d)+d B(n-1, d-1),
\end{aligned}
$$

and the result follows.

Finally, the third identity is trivial for $a=0$, so assume that $1 \leq a \leq m$ and observe that by definitions and Lemma 3(1ii) and (iii), one has

$$
\begin{aligned}
C(n, d) & =\sum_{i_{1}, \ldots, i_{d}=a+1}^{b} g_{n}\left(i_{1}, \ldots, i_{d}\right)+\sum_{j=1}^{d}\left(\sum_{i_{1}, \ldots, \hat{i}_{j}, \ldots, i_{d}=a+1}^{b} g_{n}\left(i_{1}, \ldots, i_{j-1}, a, i_{j+1} \ldots, i_{d}\right)\right) \\
& =A(n, d)+\sum_{j=1}^{d}\left(\sum_{i_{1}, \ldots, \hat{\imath}_{j}, \ldots, i_{d}=a+1}^{b} g_{n-1}\left(i_{1}-1, \ldots, i_{j-1}-1, i_{j+1}-1, \ldots, i_{d}-1\right)\right) \\
& =A(n, d)+d C(n-1, d-1),
\end{aligned}
$$

and the result follows.

Now we can complete the proof of Theorem 4 . Indeed, using twice the first identity of Lemma 5 , one gets

$$
\begin{aligned}
A(n, d+1) & =A(n, d)-(m-a) B(n-1, d)-a C(n-1, d-1), \\
d A(n-1, d) & =d A(n-1, d-1)-d(m-a) B(n-2, d-1)-d a C(n-2, d-1) .
\end{aligned}
$$

Next, the other two identities of Lemma 5 imply

$$
\begin{aligned}
& A(n, d+1)-d A(n-1, d)=A(n, d)-d A(n-1, d-1) \\
& \quad-((m-a) B(n-1, d)-(m-a) d B(n-2, d-1))-(a C(n-1, d)-a d C(n-2, d-1)) \\
& \quad=A(n, d)-d A(n-1, d-1)-(m-a) A(n-1, d)-a A(n-1, d),
\end{aligned}
$$

and the result follows.

The next result relates the sequence $\{A(n, d)\}$ to the sequence $\{f(n)\}$. 
Theorem 6 Let $n \geq k$ and $1 \leq d \leq l$, then

$$
A(n, d)=\sum_{j=0}^{d}(-1)^{j} j !\left(\begin{array}{c}
m \\
j
\end{array}\right)\left(\begin{array}{l}
d \\
j
\end{array}\right) f(n-j) .
$$

Proof. Let $D(n, d)=D_{l, m}^{a}(n, d)$ denote the right hand side of the above identity. We claim that for $n \geq k+1$ and $1 \leq d \leq l-1, D(n, d)$ satisfies the same relation (2) as $A(n, d)$ does. Indeed,

$$
\begin{aligned}
D(n-1, d) & =\sum_{j=0}^{d}(-1)^{j} j !\left(\begin{array}{c}
m \\
j
\end{array}\right)\left(\begin{array}{c}
d \\
j
\end{array}\right) f(n-1-j) \\
& =-\sum_{j=1}^{d}(-1)^{j}(j-1) !\left(\begin{array}{c}
m \\
j-1
\end{array}\right)\left(\begin{array}{c}
d \\
j-1
\end{array}\right) f(n-j)+(-1)^{d} d !\left(\begin{array}{c}
m \\
d
\end{array}\right) f(n-d-1),
\end{aligned}
$$

and

$$
\begin{aligned}
D(n-1, d-1) & =\sum_{j=0}^{d-1}(-1)^{j} j !\left(\begin{array}{c}
m \\
j
\end{array}\right)\left(\begin{array}{c}
d-1 \\
j
\end{array}\right) f(n-1-j) \\
& =-\sum_{j=1}^{d}(-1)^{j}(j-1) !\left(\begin{array}{c}
m \\
j-1
\end{array}\right)\left(\begin{array}{c}
d-1 \\
j-1
\end{array}\right) f(n-j)
\end{aligned}
$$

and hence

$$
\begin{aligned}
& D(n, d)-(m-d) D(n-1, d)-d D(n-1, d-1)=f(n)+(m-d)(-1)^{d+1} d !\left(\begin{array}{l}
m \\
d
\end{array}\right) f(n-d-1) \\
& \quad+\sum_{j=1}^{d}(-1)^{j} j !\left(\left(\begin{array}{c}
m \\
j
\end{array}\right)\left(\begin{array}{c}
d \\
j
\end{array}\right)+\frac{m-d}{j}\left(\begin{array}{c}
m \\
j-1
\end{array}\right)\left(\begin{array}{c}
d \\
j-1
\end{array}\right)+\frac{d}{j}\left(\begin{array}{c}
m \\
j-1
\end{array}\right)\left(\begin{array}{c}
d-1 \\
j-1
\end{array}\right)\right) f(n-j) \\
& \quad=f(n)+\sum_{j=1}^{d}(-1)^{j} j !\left(\begin{array}{c}
m \\
j
\end{array}\right)\left(\begin{array}{c}
d+1 \\
j
\end{array}\right) f(n-j)+(-1)^{d+1}(d+1) !\left(\begin{array}{c}
m \\
d+1
\end{array}\right) f(n-d-1)=D(n, d+1) .
\end{aligned}
$$

It follows that $D(n, d)$ (as well as $A(n, d)$ ) are defined uniquely for $n \geq k$ and $1 \leq l \leq d$ by initial values $D(k, d), D(n, 0)$, and $D(n, 1)(A(k, d), A(n, 0)$, and $A(n, 1)$, respectively). It is easy to see that for $n \geq k$ one has $A(n, 0)=D(n, 0)=f(n)$. Next, the first identity of Lemma 5 for $d=1$ gives

$$
A(n, 1)=A(n, 0)-(m-a) B(n-1,0)-a C(n-1,0)=f(n)-m f(n-1) \quad \text { for } n \geq k .
$$

On the other hand, by definition,

$$
D(n, 1)=f(n)-m f(n-1) \quad \text { for } n \geq k,
$$

and hence $A(n, 1)=D(n, 1)$. Finally, a simple combinatorial argument shows that

$$
A(k, d)=d !\left(\begin{array}{l}
l \\
d
\end{array}\right)(k-d) !-l ! m ! \quad \text { for } 1 \leq d \leq l .
$$


On the other hand,

$$
D(k, d)=\sum_{j=0}^{d}(-1)^{j} j !\left(\begin{array}{c}
m \\
j
\end{array}\right)\left(\begin{array}{c}
d \\
j
\end{array}\right)(k-j) !-l ! m !,
$$

since $f(r)=r$ ! for $1 \leq r \leq k-1$ and $f(k)=k !-l ! m !$. To prove $A(k, d)=D(k, d)$ it remains to check that

$$
\sum_{j=0}^{d}(-1)^{j} j !\left(\begin{array}{c}
m \\
j
\end{array}\right)\left(\begin{array}{l}
d \\
j
\end{array}\right)(k-j) !=d !\left(\begin{array}{l}
l \\
d
\end{array}\right)(k-d) !
$$

which follows from Lemma $\square$ below.

Finally, we are ready to prove the Main Theorem stated in Sec. 1. First of all, by Lemma B(iii), $A(n, l)=$ 0 for $n \geq k$. Hence, by Theorem 6 ,

$$
\sum_{j=0}^{l}(-1)^{j} j !\left(\begin{array}{c}
m \\
j
\end{array}\right)\left(\begin{array}{l}
l \\
j
\end{array}\right) f(n-j)=0 \quad \text { for } n \geq k
$$

or, equivalently,

$$
\sum_{j=0}^{l}(-1)^{j} j !\left(\begin{array}{c}
m \\
j
\end{array}\right)\left(\begin{array}{l}
l \\
j
\end{array}\right) x^{j} f(n-j) x^{n-j}=0 \quad \text { for } n \geq k .
$$

As was already mentioned, $f(r)=r$ ! for $1 \leq r \leq k-1$, therefore, summing over $n \geq k$ yields

$$
\sum_{j=0}^{l}(-1)^{j} j !\left(\begin{array}{c}
m \\
j
\end{array}\right)\left(\begin{array}{l}
l \\
j
\end{array}\right) x^{j}\left(F_{l, m}^{a}(x)-\sum_{i=0}^{k-j-1} x^{i} i !\right)=0 .
$$

Recall that the rook polynomial of the rectangular $s \times t$ board, $s \leq t$, satisfies relation

$$
R_{s, t}(x)=\sum_{j=0}^{s} j !\left(\begin{array}{l}
t \\
j
\end{array}\right)\left(\begin{array}{l}
s \\
j
\end{array}\right) x^{j}
$$

(see [R1, Ch. 7.4]). Hence, (3) is equivalent to

$$
F_{l, m}^{a}(x) R_{\lambda, \mu}(-x)=\sum_{j=0}^{l}(-1)^{j} j !\left(\begin{array}{c}
m \\
j
\end{array}\right)\left(\begin{array}{l}
l \\
j
\end{array}\right) x^{j} \sum_{i=0}^{k-j-1} x^{i} i !=\sum_{r=0}^{k-1} x^{r} r ! \sum_{j=0}^{r}(-1)^{j} \frac{\left(\begin{array}{l}
l \\
j
\end{array}\right)\left(\begin{array}{c}
m \\
j
\end{array}\right)}{\left(\begin{array}{l}
r \\
j
\end{array}\right)} .
$$

Let us divide the external sum in the above expression into three parts: the sum from $r=0$ to $\lambda-1$, the sum from $r=\lambda$ to $\mu-1$, and the sum from $r=\mu$ to $k-1$. By Lemma 7 below, the third sum vanishes, while the second sum is equal to

$$
\sum_{r=\lambda}^{\mu-1} x^{r} r !(-1)^{\lambda} \frac{(r-\lambda) !(k-r-1) !}{(\mu-r-1) ! r !}
$$

and the first expression of the Main Theorem follows. The second expression is obtained easily from (3) and relation between rook polynomials and Laguerre polynomials given in Sec. 1.

It remains to prove the following technical result, which is apparently known; however, we failed to find a reference to its proof, and decided to present a short proof inspired by the brilliant book [PWZ]. 
Lemma 7 Let $1 \leq s \leq t$ and let

$$
M(s, t)=\sum_{i=0}^{s}(-1)^{i} \frac{\left(\begin{array}{l}
s \\
i
\end{array}\right)\left(\begin{array}{l}
t \\
i
\end{array}\right)}{\left(\begin{array}{l}
n \\
i
\end{array}\right)}
$$

Then:

$$
M(s, t)=\left\{\begin{array}{lr}
\frac{\left(\begin{array}{c}
n-t \\
s
\end{array}\right)}{\left(\begin{array}{l}
n \\
s
\end{array}\right)} & \text { if } n \geq s+t, \\
0 & \text { if } t \leq n \leq s+t-1, \\
(-1)^{s} \frac{\left(\begin{array}{c}
s+t-n-1 \\
s
\end{array}\right)}{\left(\begin{array}{l}
n \\
s
\end{array}\right)} & \text { if } s \leq n \leq t-1 .
\end{array}\right.
$$

Proof. Direct check reveals that $M(s, t)$ is a hypergeometric series; to be more precise,

$$
M(s, t)={ }_{2} F_{1}\left[\begin{array}{l}
-t,-s \\
-n
\end{array} ; 1\right] .
$$

Since $-s$ is a nonpositive integer, the Gauss formula applies (see [PWZ, Ch. 3.5]), and we get

$$
M(s, t)=\lim _{z \rightarrow n} \frac{\Gamma(-z+t+s) \Gamma(-z)}{\Gamma(t-z) \Gamma(s-z)} .
$$

Recall that

$$
\Gamma(x) \Gamma(1-x)=\frac{\pi}{\sin \pi x} .
$$

If $n \geq s+t$, we apply (4) for $x=-z+t+s, x=t-z, x=s-z, x=-z$, and get

$$
M(s, t)=-\frac{\Gamma(n-t+1) \Gamma(n-s+1)}{\Gamma(n-t-s+1) \Gamma(n+1)} \lim _{z \rightarrow n} \frac{\sin \pi(t-z) \sin \pi(s-z)}{\sin \pi z \sin \pi(t+s-z)}=\frac{\left(\begin{array}{c}
n-t \\
s
\end{array}\right)}{\left(\begin{array}{l}
n \\
s
\end{array}\right)} .
$$

If $t \leq n \leq s+t-1$, we apply (4) for $x=t-z, x=s-z, x=-z$, and get

$$
M(s, t)=-\frac{\Gamma(n-t+1) \Gamma(n-s+1) \Gamma(s+t-n)}{\Gamma(n+1)} \lim _{z \rightarrow n} \frac{\sin \pi(t-z) \sin \pi(s-z)}{\sin \pi z}=0 .
$$

Finally, if $s \leq n \leq t-1$, we apply (4) for $x=s-z, x=-z$, and get

$$
M(s, t)=-\frac{\Gamma(s+t-n) \Gamma(n-s+1)}{\Gamma(t-n) \Gamma(n+1)} \lim _{z \rightarrow n} \frac{\sin \pi(s-z)}{\sin \pi z}=(-1)^{s} \frac{\left(\begin{array}{c}
s+t-n-1 \\
s
\end{array}\right)}{\left(\begin{array}{l}
n \\
s
\end{array}\right)} .
$$

\section{Concluding remarks}

Observe first, that according to the Main Theorem, $F_{l, m}^{a}(x)$ does not depend on $a$; in other words, $\left|S_{n}\left(P_{l, m}\right)\right|=$ $\left|S_{n}\left(\sigma^{a} P_{l, m}\right)\right|$ for any $a$. We obtained this fact as a consequence of lengthy computations. A natural question would be to find a bijection between $S_{n}\left(P_{l, m}\right)$ and $S_{n}\left(\sigma^{a} P_{l, m}\right)$ that explains this phenomenon. 
Second, it is well known that rook polynomials (or the corresponding Laguerre polynomials) are related to permutations with restricted positions, see [R1, Ch.7,8]. Laguerre polynomials also arise in a natural way in the study of generalized derangements (see [EZ] and references therein). It is tempting to find a combinatorial relation between permutations with restricted positions and permutations avoiding maximal parabolic subgroups, which could explain the occurrence of Laguerre polynomials in the latter context.

Finally, one can consider permutations avoiding nonmaximal parabolic subgroups of $S_{k}$. The first natural step would be to treat the case of subgroups generated by $k-3$ simple transpositions. It is convenient to denote by $P_{l_{1}, l_{2}, l_{3}}$ (with $l_{1}+l_{2}+l_{3}=k$ ) the subgroup of $S_{k}$ generated by all the simple transpositions except for $s_{l_{1}}$ and $s_{l_{1}+l_{2}}$; further on, we set $f_{l_{1}, l_{2}, l_{3}}(n)=\left|S_{n}\left(P_{l_{1}, l_{2}, l_{3}}\right)\right|$, and $F_{l_{1}, l_{2}, l_{3}}(x)=\sum_{n \geq 0} f_{l_{1}, l_{2}, l_{3}}(n) x^{n}$. It is easy to see that $F_{l_{1}, l_{2}, l_{3}}(x)=F_{l_{3}, l_{2}, l_{1}}(x)$, so one can assume that $l_{1} \leq l_{3}$. This said, the main result of [BDPP] can be formulated as follows: let $k \geq 3$, then

$$
F_{1,1, k-2}(x)=\sum_{r=1}^{k-3} x^{r} r !+\frac{(k-3) ! x^{k-4}}{2}\left(1-(k-1) x-\sqrt{1-2(k-1) x+(k-3)^{2} x^{2}}\right) .
$$

To the best of our knowledge, this is the only known instance of $F_{l_{1}, l_{2}, l_{3}}(x)$. It is worth noting that even in this, simplest case of nonmaximal parabolic subgroup, the generating function is no longer rational.

\section{References}

[AR] R. Adin and Yu. Roichman, Shape avoiding permutations, (1999), preprint math.CO/9912119.

[BDPP] E. Barcucci, A. Del Lungo, E. Pergola, and R. Pinzani, Permutations avoiding an increasing number of length-increasing forbidden subsequences, Discrete Math. Theor. Comput. Sci. 4 (2000), 31-44.

[FZ] D. Foata and D. Zeilberger, Laguerre polynomials, weighted derangements, and positivity, SIAM J. Discr. Math. 1 (1988), 425-433.

[IL] M. Ismail and X. Li, Bounds on the extreme zeros of orthogonal polynomials, Proc. AMS 115 (1992), 131-140

[Kn] D. Knuth, The Art of Computer Programming, vol. 1, Addison Wesley, Reading, MA, 1968.

[LS] V. Lakshmibai and B. Sandhya, Criterion for smoothness of Schubert varieties in $\mathrm{Sl}(n) / B$, Proc. Indian Acad. Sci. 100 (1990), 45-52.

[Ma] T. Mansour, Permutations containing and avoiding certain patterns, Proc. 12th Conference on Formal Power Series and Algebraic Combinatorics (Moscow, 2000), 2000.

[PWZ] M. Petkovšek, H. Wilf, and D. Zeilberger, A=B, A. K. Peters, Wellesley, MA, 1996.

[Ri] J. Riordan, An Introduction to Combinatorial Analysis, Wiley, New York, 1967.

[SS] R. Simion and F. Schmidt, Restricted permutations, Europ. J. Comb. 6 (1985), 383-406.

[St] R. Stanley, Enumerative Combinatorics, vols 1, 2, Cambridge University Press, Cambridge, 1997, 1999.

[Sz] G. Szego, Orthogonal Polynomials, AMS, Providence, RI, 1967. 
